$\mathrm{e}^{+} \mathrm{e}^{-}$Collisions from Phi to Psi 2013 (PHIPSI2013)

International Journal of Modern Physics: Conference Series

Vol. 35 (2014) 1460439 (5 pages)

(C) The Author

DOI: $10.1142 /$ S2010194514604396

\title{
RECENT RESULTS ON $\tau$ LEPTON PHYSICS FROM BELLE
}

\author{
SIMON EIDELMAN \\ for the Belle Collaboration \\ Budker Institute of Nuclear Physics SB RAS, Novosibirsk 630090, Russia \\ and Novosibirsk State University, Novosibirsk 630090, Russia \\ Published 18 December 2014
}

\begin{abstract}
We report recent results on $\tau$ lepton physics obtained with the Belle detector. They include a search for lepton-flavor-violating decays $\tau \rightarrow l h h^{\prime}$, a high-precision measurement of the $\tau$ lepton lifetime and a study of the hadronic decays with the $K_{S}^{0}$ in the final state.
\end{abstract}

Keywords: Tau lepton.

PACS Numbers: 13.35.Dx, 13.66.De

\section{Introduction}

Belle, ${ }^{1}$ the experiment at the KEKB $e^{+} e^{-}$collider at KEK ( $B$-factory) ${ }^{2}$ collected an integrated luminosity $\simeq 1 \mathrm{ab}^{-1}$ at the peak of the $\Upsilon(4 S)$ resonance and nearby. At this energy the cross section of the $\tau^{+} \tau^{-}$pair production is about $0.92 \mathrm{nb}$, so that the collected luminosity corresponds to about a billion of $\tau^{+} \tau^{-}$pairs produced. Thus, the $B$-factory is also a $\tau$ lepton factory providing a possibility of high-precision studies of the Standard Model (SM) $\tau$ physics and a search for very rare or forbidden in SM decay modes. In this talk we briefly describe three recent analyses related to $\tau$ leptons.

\section{Search for Lepton Flavor Violation}

The probability of lepton-flavor-violating (LFV) decays of charged leptons is extremely small in $\mathrm{SM}, \mathcal{B}(\tau \rightarrow \ell \gamma) \sim\left(\frac{\Delta m_{\nu}^{2}}{m_{W}^{2}}\right)^{2}<10^{-54}$. However, many models beyond the SM predict LFV decays with the branching fractions up to $\sim 10^{-8}$, so LFV observation is a clear signature of New Physics (NP). A $\tau$ lepton is an excellent laboratory to search for the LFV decays: its large mass results in enhanced

This is an Open Access article published by World Scientific Publishing Company. It is distributed under the terms of the Creative Commons Attribution 3.0 (CC-BY) License. Further distribution of this work is permitted, provided the original work is properly cited. 


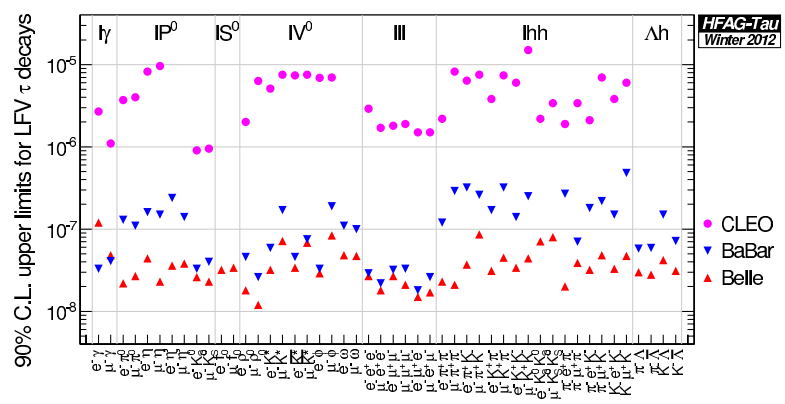

Fig. 1. Summary of the LFV searches at CLEO, BaBar and Belle

couplings to new particles and the large number of LFV decay modes, which test various NP models. ${ }^{3}$

Belle performed a search for LFV decays of the $\tau$ lepton in which 14 modes were studied with $854 \mathrm{fb}^{-1}$ of integrated luminosity corresponding to $782 \times 10^{6} \tau$ pairs produced: $8 \mathrm{LFV} \tau^{-} \rightarrow \ell^{-} h^{+} h^{--}$and 6 lepton-number-violating $\tau^{-} \rightarrow \ell^{+} h^{-} h^{-}$ decays. Here $\ell$ stands for $e$ or $\mu$ and $h$ for $\pi$ or $K$.

After blind analysis and thorough estimation of the possible background sources, one event in the signal region was found for $\tau^{-} \rightarrow \mu^{+} \pi^{-} \pi^{-}$and $\tau^{-} \rightarrow \mu^{-} \pi^{+} K^{-}$and there were no events for the other 12 modes. For all modes the number of observed signal events agrees with the number of expected background events, so that the upper limits are set on the corresponding branching fractions. The obtained upper limits are $\mathcal{B}\left(\tau \rightarrow \ell h h^{\prime}\right)<(2.0 \div 8.6) \times 10^{-8}$ at $90 \% \mathrm{CL}$ and are the most stringent for such decays. ${ }^{4}$

In total, 48 different LFV decay modes were studied at Belle of which 46 modes were analysed with almost full Belle statistics $\left(\sim 1 \mathrm{ab}^{-1}\right)$ and the world best upper limits were obtained. A full statistics study of $\tau \rightarrow \mu(e) \gamma$ is in progress and will be completed soon. Figure 1 summarizes the results of the LFV searches performed at CLEO, BaBar and Belle. ${ }^{5}$

\section{Measurement of $\tau$ Lepton Lifetime}

Knowledge of the $\tau$ Lepton Lifetime is essential for tests of lepton universality. Currently its world average is completely dominated by the LEP experiments and CLEO. ${ }^{6}$ Belle performed a new analysis with $711 \mathrm{fb}^{-1}$ or $650 \times 10^{6} \tau$ pairs using events in which both $\tau^{ \pm}$leptons decay to $\pi^{ \pm} \pi^{+} \pi^{-} \bar{\nu}_{\tau} \cdot{ }^{7}$ The $\tau$ lepton momentum direction is determined with two-fold ambiguity in the center-of-mass system (CMS), therefore we use the average axis of the two. The asymmetric-energy layout allows us to determine the $\tau^{+} \tau^{-}$production point in the laboratory system independently of the interaction point. Moreover, one can also perform a CPT test from the separately measured $\tau^{-}$and $\tau^{+}$lifetimes. Analysis of systematic effects (Table 1) shows that the dominant source is SVD alignment. 
Table 1. Systematic uncertainties for the $\tau$ lifetime measurement

\begin{tabular}{lc}
\hline Source & $\Delta c \tau(\mu m)$ \\
\hline SVD alignment & 0.090 \\
Fit range & 0.020 \\
ISR and FSR description & 0.018 \\
Beam energy & 0.016 \\
Background contribution & 0.010 \\
$\tau$-lepton mass accuracy & 0.009 \\
\hline Total & 0.096 \\
\hline
\end{tabular}

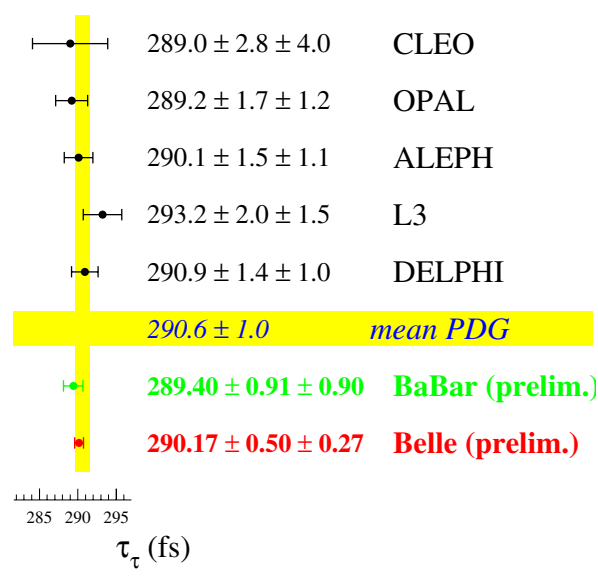

Fig. 2. Comparison of the Belle result for the $\tau$ lifetime with the previous measurements

Our result for the lifetime is

$$
\left.\left.\tau_{\tau}=(290.17 \pm 0.50 \text { (stat. }) \pm 0.33 \text { (syst. }\right)\right) \times 10^{-15} \mathrm{~s},
$$

consistent with the world average of $(290.6 \pm 1.0) \times 10^{-15} \mathrm{~s}$ and 1.6 times more precise. We compare the Belle result with the previous measurements in Fig. 2. The most accurate $\tau$ lifetime determination performed with the DELPHI detector has three times worse accuracy. ${ }^{8}$ From the separately measured lifetimes of the $\tau^{-}$and $\tau^{+}$we perform their world first comparison:

$$
\left|\tau_{\tau^{+}}-\tau_{\tau^{-}}\right| / \tau_{\text {average }}<7.0 \times 10^{-3} \text { at } 90 \% \mathrm{CL}
$$

\section{4. $\tau$ Lepton Decays with $K_{S}^{0}$}

Decays with kaons in the final state provide a lot of interesting information on $\tau$ lepton spectral functions, the CKM matrix element $\left|V_{u s}\right|$, properties of various $K^{*}$ mesons and have other important applications. However, they are still rather badly studied. 

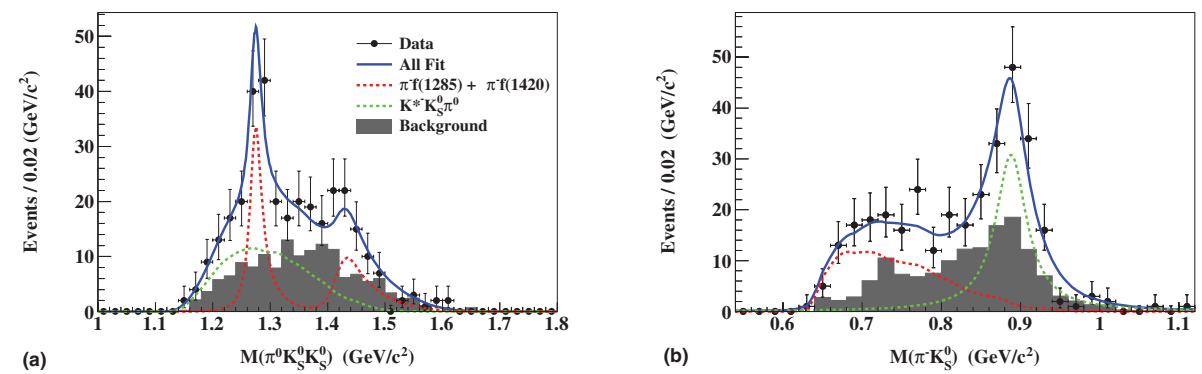

Fig. 3. Dynamics of the $\tau^{-} \rightarrow \pi^{-} K_{S}^{0} K_{S}^{0} \pi^{0} \nu_{\tau}$ decay: (a) - distribution of the $K_{S}^{0} K_{S}^{0} \pi^{0}$ mass, (b) - distribution of the $K_{S}^{0} \pi^{-}$mass

A data sample with integrated luminosity of $669 \mathrm{fb}^{-1}$ corresponding to $616 \times 10^{6}$ pairs was used to study the inclusive decay $\tau^{-} \rightarrow K_{S}^{0} X^{-} \nu_{\tau}$ as well as 6 exclusive modes: $\pi^{-} K_{S}^{0} \nu_{\tau}, K^{-} K_{S}^{0} \nu_{\tau}, \pi^{-} K_{S}^{0} K_{S}^{0} \nu_{\tau}, \pi^{-} K_{S}^{0} \pi^{0} \nu_{\tau}, K^{-} K_{S}^{0} \pi^{0} \nu_{\tau}$ and $\pi^{-} K_{S}^{0} K_{S}^{0} \pi^{0} \nu_{\tau}$. The results of the analysis are presented in Table 2 . In all cases the obtained values of the branching fractions are consistent with the other measurements and their precision is significantly higher than in the experiments prior to the $B$-factories. This is the first measurement of the decay modes $\tau^{-} \rightarrow K^{-} K_{S}^{0} \nu_{\tau}$ and $\tau^{-} \rightarrow K^{-} K_{S}^{0} \pi^{0} \nu_{\tau}$ performed at the $B$-factory. For the decay mode with the smallest branching fraction, $\tau^{-} \rightarrow \pi^{-} K_{S}^{0} K_{S}^{0} \pi^{0} \nu_{\tau}$, our result is consistent with that of $\mathrm{BaBar}^{9}$ and has better precision.

In addition, we have also studied the internal dynamics for various decays. In particular, in the decay mode $\tau^{-} \rightarrow \pi^{-} K_{S}^{0} K_{S}^{0} \pi^{0} \nu_{\tau}$ the $f_{1}(1285) \pi^{-} \nu_{\tau}(5.9 \sigma)$ and $K^{*-}(892) K_{S}^{0} \nu_{\tau}$ intermediate structures are observed, as well as indication of the $f_{1}(1420) \pi^{-} \nu_{\tau}(2.7 \sigma)$ mechanism is seen, as it is clear from Fig. 3. The corresponding branchings are

$$
\mathcal{B}\left(\tau^{-} \rightarrow\left(f_{1}(1285) \rightarrow K_{S}^{0} K_{S}^{0} \pi^{0}\right) \pi^{-} \nu_{\tau}\right)=(0.74 \pm 0.12 \pm 0.07) \times 10^{-5}
$$

and

$$
\mathcal{B}\left(\tau^{-} \rightarrow\left(K^{*-} \rightarrow K_{S}^{0} \pi^{-}\right) K_{S}^{0} \pi^{0} \nu_{\tau}\right)=(1.06 \pm 0.15 \pm 0.09) \times 10^{-5}
$$

Table 2. Branching fractions

\begin{tabular}{c|c|cccccc}
\hline Mode & $K_{S}^{0} X^{-}$ & $\pi^{-} K_{S}^{0}$ & $K^{-} K_{S}^{0}$ & $\pi^{-} K_{S}^{0} \pi^{0}$ & $K^{-} K_{S}^{0} \pi^{0}$ & $\pi^{-} K_{S}^{0} K_{S}^{0}$ & $\pi^{-} K_{S}^{0} K_{S}^{0} \pi^{0}$ \\
\hline$N^{\text {data }}$ & $397806 \pm 631$ & $157836 \pm 541$ & $32701 \pm 295$ & $26605 \pm 208$ & $8267 \pm 109$ & $6684 \pm 96$ & $303 \pm 33$ \\
$\varepsilon_{\text {det }}(\%)$ & 9.66 & 7.09 & 6.69 & 2.65 & 2.19 & 2.47 & 0.82 \\
$\frac{N^{\text {bg }}}{N^{\text {data }}}(\%)$ & $4.20 \pm 0.46$ & $8.86 \pm 0.05$ & $3.55 \pm 0.07$ & $5.60 \pm 0.10$ & $2.43 \pm 0.10$ & $7.89 \pm 0.24$ & $11.60 \pm 1.60$ \\
$\left(\frac{\Delta \mathcal{B}}{\mathcal{B}}\right)_{\text {syst }}(\%)$ & 2.4 & 2.5 & 4.0 & 3.9 & 5.2 & 4.4 & 8.1 \\
\hline
\end{tabular}




\section{Summary}

- Belle collected the world largest data sample of $\sim 1 \mathrm{ab}^{-1}\left(N_{\tau \tau} \simeq 10^{9}\right)$ near the $\Upsilon(4 S)$ opening a new era in precise $\tau$ physics

- 48 different LFV modes were studied and upper limits on their branching fractions $\mathcal{B} \sim \mathcal{O}\left(10^{-8}\right)$ were obtained

- With $711 \mathrm{fb}^{-1}$ the $\tau$ lifetime and upper limit on the relative lifetime difference between $\tau^{+}$and $\tau^{-}$have been measured using a new method:

$\tau_{\tau}=(290.17 \pm 0.50$ (stat.) \pm 0.33 (syst.) $) \times 10^{-15} \mathrm{~s}$, $\left|\tau_{\tau^{+}}-\tau_{\tau^{-}}\right| / \tau_{\text {average }}<7.0 \times 10^{-3}$ at $90 \%$ CL.

- The branching fractions for six $\tau$ decay modes with $K_{S}^{0}$ and for the inclusive decay $\tau^{-} \rightarrow K_{S}^{0} X^{-} \nu_{\tau}$ have been measured. The unfolded invariant mass spectra have been obtained for the $\tau^{-} \rightarrow K_{S}^{0} \pi^{-} \pi^{0} \nu_{\tau}$ and $\tau^{-} \rightarrow K_{S}^{0} K^{-} \pi^{0} \nu_{\tau}$ modes, for the latter $f_{1}(1285) \pi^{-} \nu_{\tau}$ and $K^{*-}(892) K_{S}^{0} \nu_{\tau}$ mechanisms were observed

There are many other ongoing studies of hadronic $\tau$ decays at Belle, which include a study of the spectral functions of $\tau^{-} \rightarrow \pi^{-} \pi^{-} \pi^{+} \pi^{0} \nu_{\tau}$ and $\tau^{-} \rightarrow \pi^{-} \pi^{0} \pi^{0} \nu_{\tau}$ decays, a search for CP violation in $\tau^{-} \rightarrow K^{-} \pi^{-} \pi^{+} \nu_{\tau}$ decay, measurements of the branching fractions of $\tau^{-} \rightarrow \pi^{-} \geq 2 \pi^{0} \nu_{\tau}$ and $\tau^{-} \rightarrow h_{1}^{-} h_{2}^{-} h_{3}^{+} \nu_{\tau}, h_{1,2,3}=\pi, K$ decays, a search for 2nd class currents in $\tau^{-} \rightarrow \eta \pi^{-} \nu_{\tau}$ and $\tau^{-} \rightarrow \eta^{\prime} \pi^{-} \nu_{\tau}$ decays.

\section{Acknowledgments}

The author is grateful to the Workshop organizers for the interesting program and kind hospitality, to Novosibirsk State University for support and to his Belle colleagues for useful discussions. This work is supported by the Ministry of Education and Science of the Russian Federation, the RFBR grant 12-02-01032 and the DFG grant HA $1457 / 9-1$.

\section{References}

1. A. Abashian et al., Nucl. Instr. Meth. A 479, 117 (2002); see also the detector session in J. Brodzicka et al., Prog. Theor. Exp. Phys. 2012, 04D001 (2012).

2. S. Kurokawa and E. Kikutani, Nucl. Instr. Meth. A 499, 1 (2003), and other papers included in this volume; T. Abe et al., Prog. Theor. Exp. Phys. 2013, 03 A001 (2013) and following articles up to 03A011.

3. A. Stahl, Physics with Tau Leptons, (Springer-Verlag, Berlin, Heidelberg, 2000).

4. Belle Collab. (Y. Miyazaki et al.), Phys. Lett. B 719, 346 (2013).

5. K. Hayasaka, Nucl. Phys. Proc. Suppl. 225-227, 169 (2012).

6. Particle Data Group (J. Beringer et al.), Phys. Rev. D 86, 010001 (2012).

7. Belle Collab. (K. Belous et al.), arXiv:1310.8503.

8. DELPHI Collab. (J. Abdallah et al.), Eur. Phys. J. C 36, 283 (2004).

9. BaBar Collab. (J.P. Lees et al.), Phys. Rev. D 86, 092013 (2012). 